\title{
Singing Meridian Study_-Theoretical and Practical Study for Applying Meridian Theory into Singing Pronunciation
}

\author{
Zhao Ling ${ }^{1,2}$ \\ 1. Jiaozuo Teachers College, No. 998, Shanyang District, Jiaozuo City 454002, Henan Province, China \\ 2. Shan Yang District, Guangchang Huayuan (Square Garden) Apartment, Buiding A 03-3 ,Jiaozuo City 454002, Henan Province, \\ China
}

\begin{abstract}
The author created singing meridian theory system and practice method for the purpose of tapping singing control system potential of human body to the greatest extent, developing controlling function of meridian system in all-round way, integrating blood circulating improvement function with singing organic function through acupoint stimulation , and improving singing voicing and disease prevention capabilities. The thesis argued that both singing motion method and disease rehabilitation treatment are final results of organ interactions of meridian system. Argument Methods: The author applies meridian theory and acupoint stimulation method into singing teaching research. Adopting experiment of stimulating acupoint with fixed acupoint, position, nature and quantity while making singing pronunciation, the author studies acupoints of Yifeng, Yintang and Zhongfu, etc. to identify singing pronunciation changes under acupoint stimulation. The method pioneers experiment research of singing controlled by meridian system, proposes singing meridian control system theory and expands singing meridian controlling function of heal care and rehabilitation treatment. Through strengthening of organic connections between different systems such as body, meridians and other functional organs of the body, singing meridian control system constitute meridian theory and training mechanism combing "breath, blood, sound”, and aims to fully taps the potential of singing, avoids normal problems, shortens learning cycle and improves the efficiency of the singing and treatment. In order to popularize and learn singing and health care knowledge through acupoint stimulation, a new systematic and scientific way of learning and practice is provided to widen the application field of singing meridian theory.
\end{abstract}

Key words: Singing teaching, meridian theory, control system, acupoint stimulation.

\section{Proposal of Singing Meridian Theory}

In 1992, I applied meridian theory and acupoint stimulation technique [4] into singing study and training process, and proved to be very effective. To prove this effect, I made some scientific experiment to verify the strengthened effect of acupoint stimulation in singing and rehabilitation process. Based on scientific experiment results, I created singing meridian control system theory and published a paper in 1996. In 2006, I published my original academic monograph Singing Monograph Study. Then in 2014 I

Correspondence author: Zhao Ling, professor, research fields: music therapy, traditional Chinese medicine keeping of in good health and treatment. E-mail: 807649192@qq.com. published Singing Meridian Science (Volume one: Theory and practice for applying acupuncture stimulation into singing pronunciation; Volume two: Singing teaching and practice combining breath, blood and song), and created singing meridian science [1].

\section{Scientific Theoretic Basis for Singing Meridian Theory}

2.1 Body System and Meridian System Are Material Basis for Forming Singing “Instrument” System

By applying meridian system and acupuncture manipulating technique to singing pronunciation control and adjustment process, study and training 
effect is sped up and the whole singing learning process [1] is optimized. This study method gives full expression to holism in Chinese traditional medicine [8] and provides scientific theoretical basis and feasible practical [2] grounds for creating singing meridian science theory. Through organic integrating of singing theoretical practice and meridian theoretical practice, singing meridian control system theory [1] which constitutes core parts of singing meridian theory is founded [2].

\subsection{Acupoint Stimulation Is an Effective Method for Enhancing Speed Changes of Singing Pronunciation}

Singing meridian control system theory [1] advances traditional singing in memorizing method, changing passive auditory reliance memorizing method to active kinetic sensing singing method.

\subsection{Training Method of Integrating "Breath, Blood} and Tone" [1]

Traditional singing learning mainly adopts descending pathway method [6] in which singing consciousness governs motor center. The new method co-works, integrates and overlaps with the ascending pathway method to govern and create singing consciousnesses through stimulating acupoint sensory, and hence intensifies singing sensory memorizing function and ushers in double multiplying high-efficient instant memorizing function. With such method, singing kinetic sensory and acupoint sensory feelings works together, and forms singing learning and disease rehabilitation training method integrating "breath, blood and sound”. It also created an unprecedented singing meridian control training method [2].

\subsection{Biological Acoustics Theoretical Basis for Singing} Meridian Control System

Acupoint sensory feelings coordinate with singing dynamic movement, making organ system change in terms of force structure movement morphology, while at the same time cause organ voicing movement structure to be changed, leading to changes of voicing in terms of harmonic series and amplitude [2], and hence cause changes of voice.

\section{Experiment of Applying Acupoint Stimulation to Pronunciation Organic Function Training}

To prove aftereffect of acupoint stimulation in singing pronunciation, we verified through relevant experiment and came to the conclusion that stimulating acupoint can work on singing pronunciation with optimistic [1], effective and everlasting effect.

\subsection{Experiment of Voice Fatique Resistance through Stimulating YiFeng Acupoint [7]}

The experiment of singing while stimulating Yifeng acupoint proves that stimulating Yifeng acupoint can resist voice fatigue (Table 1 ).

Table 1 Fatigue status of experiment for three groups (press, acupuncture, and no stimulation).

\begin{tabular}{|c|c|c|c|c|}
\hline $\begin{array}{l}\text { Experiment groups } \\
\text { (concentration changes) }\end{array}$ & $\begin{array}{l}\text { Cases } \\
\text { (Male/Female) }\end{array}$ & $\begin{array}{l}\text { Fatigue occurrence } \\
\text { (Peson No./Time) }\end{array}$ & $\begin{array}{l}\text { Feeling indicator } \\
\text { ((Dry, moisturized) }\end{array}$ & $\begin{array}{l}\text { Objective indicator } \\
\text { (saliva quantity) }\end{array}$ \\
\hline Group A & $7 / 10$ & 0 person $/ 8 \mathrm{~min}$ & moisturized & $\begin{array}{l}\text { Saliva is a little more than } \\
\text { that during practice. }\end{array}$ \\
\hline (Pressed) & & 0 person/10min & moisturized & Saliva is light and thin. \\
\hline Group B & $7 / 10$ & 0 person/20min & moisturized & $\begin{array}{l}\text { Saliva is the same as that } \\
\text { before practice }\end{array}$ \\
\hline \multirow[t]{2}{*}{ (Stimulated) } & & & & No obvious change \\
\hline & & 0 person/40min & moisturized & Saliva is thin and light. \\
\hline Group C & $7 / 9$ & 9 person/3min & dry & Saliva is plenty and thick. \\
\hline (No stimulation) & & 7 person $/ 5 \mathrm{~min}$ & dry & Saliva is plenty and thick \\
\hline
\end{tabular}




\subsection{Experiment of Proving that Stimulating Yifeng} Acupoint Can Help Expand Singing Range

The experiment of Singing while stimulating Yifeng acupoint proves that stimulating Yifeng acupoint can rapidly expand singing range (Table 2).

3.3 Experiment to Prove that Stimulating ZhongFu Acupoint [7] Can Help Strengthen Singing Breathing Function

Stimulating Zhongfu acupoint while singing proves that pressing Zhongfu acupoint can enhance breathing control function (Table 3).
3.4 Stimulate Neiguan Acupoint [7] to Enhance the Function of Voicing Amplitude and Frequency

Singing while stimulating Neiguan acupoint proves that stimulating Neiguan acupoint can cause changes of amplitude and frequency while singing (Table 4).

3.5 The Experiment to Prove Yintang Acupoint [7] Simulation Is Beneficial to Singing Voicing Expansion and Unification

The experiment to stimulate Instant appoint proves that Yintang acupoint stimulation can expand voice range (Table 5).

Table 2 The change of range of stimulus Yifeng before and after.

\begin{tabular}{|c|c|c|c|c|c|c|c|c|}
\hline \multicolumn{5}{|c|}{ Female } & \multicolumn{4}{|c|}{ Male } \\
\hline $\begin{array}{l}\text { Range } \\
\text { (Unpressed) }\end{array}$ & $\begin{array}{l}\text { Range } \\
\text { (Pressed) }\end{array}$ & $\begin{array}{l}\text { Range } \\
\text { (Unpressed } \\
\text { Semitone No.) }\end{array}$ & $\begin{array}{l}\text { Extension range } \\
\text { (Pressed Semitone } \\
\text { No.) }\end{array}$ & $\begin{array}{l}\text { Training } \\
\text { (Time) }\end{array}$ & $\begin{array}{l}\text { Range } \\
\text { (Unpressed) }\end{array}$ & $\begin{array}{l}\text { Range } \\
\text { (Pressed) }\end{array}$ & $\begin{array}{l}\text { Range } \\
\text { (Unpressed } \\
\text { Semitone No.) }\end{array}$ & $\begin{array}{l}\text { Extension range } \\
\text { (Pressed } \\
\text { Semitone No.) }\end{array}$ \\
\hline${ }^{\#} c^{1}-e^{2}$ & $b-{ }^{\#} g^{2}$ & 16 & 24 & $3 \min$ & $d^{1}-f^{2}$ & $a-a^{2}$ & 16 & 25 \\
\hline${ }^{\#} \mathrm{c}^{1}-\mathrm{f}^{2}$ & $a-a^{2}$ & 17 & 25 & $3 \mathrm{~min}$ & $a-f^{2}$ & ${ }^{\#} \mathrm{f}-\mathrm{a}^{2}$ & 21 & 28 \\
\hline$c^{1}-e^{2}$ & $a-a^{2}$ & 17 & 25 & $3 \mathrm{~min}$ & $c^{1}-e^{2}$ & $a-{ }^{\#} g^{2}$ & 17 & 24 \\
\hline$c^{1}-e^{2}$ & $a^{\#}-g^{2}$ & 17 & 24 & $3 \mathrm{~min}$ & $c^{1}-d^{2}$ & $a-{ }^{\#} g^{2}$ & 15 & 24 \\
\hline$c^{1}-{ }^{-} f^{2}$ & $a-{ }^{b} b^{2}$ & 19 & 26 & $3 \mathrm{~min}$ & $e^{1}-a^{2}$ & $a-c^{3}$ & 18 & 28 \\
\hline$c^{1}-e^{2}$ & $a-{ }^{b} b^{2}$ & 17 & 26 & $3 \mathrm{~min}$ & $c^{1}-{ }^{\#} \mathrm{f}^{2}$ & $a-b^{2}$ & 19 & 27 \\
\hline$c^{1}-\# f^{2}$ & $a-c^{3}$ & 19 & 28 & $3 \mathrm{~min}$ & $c^{1}-{ }^{\#} f^{2}$ & $a-b^{2}$ & 19 & 27 \\
\hline$c^{1}-{ }^{-} f^{2}$ & $a-c^{2}$ & 19 & 28 & $3 \mathrm{~min}$ & $c^{1}-{ }^{\#} f^{2}$ & $a-b^{2}$ & 19 & 27 \\
\hline
\end{tabular}

Mean value $=17.933$ 26, Standard deviation $=1.4347$ 1.4951, $\mathrm{df}=15 \mathrm{t}=5.6708 \mathrm{E}-16$ t $0.001 / 2=2.947$.

Table 3 The change of pronunciation time of stimulation Zhongfu before and after.

\begin{tabular}{llllll}
\hline & \multicolumn{2}{c}{ Female } & & \multicolumn{2}{c}{ Male } \\
\hline $\begin{array}{l}\text { Lasting time } \\
\text { (Unpressed) }\end{array}$ & $\begin{array}{l}\text { Lasting time } \\
\text { (Pressed) }\end{array}$ & $\begin{array}{l}\text { Pronunciation Time } \\
\text { differences }\end{array}$ & $\begin{array}{l}\text { Lasting time } \\
\text { (Unpressed) }\end{array}$ & $\begin{array}{l}\text { Lasting time } \\
\text { (Pressed) }\end{array}$ & $\begin{array}{l}\text { Pronunciation time } \\
\text { differences }\end{array}$ \\
9.8 & 12.8 & 3 & 10.3 & 26.1 & 15.8 \\
25.8 & 27 & 1.2 & 10.1 & 14 & 3.9 \\
22.8 & 25.8 & 3 & 10.2 & 16.3 & 6.1 \\
22.9 & 25.8 & 2.9 & 17.3 & 22.7 & 5.4 \\
15.8 & 21.8 & 6 & 23 & 35 & 12 \\
12 & 18.6 & 6.6 & 19.3 & 22.2 & 3.9 \\
19.7 & 25 & 5.3 & 2 & 24 & 3 \\
17 & 19.8 & 2.8 & 21.3 & 24.3 & 6.8 \\
14.2 & 15.6 & 14 & 13 & 19.8 & 8 \\
13.3 & 24.8 & 11.5 & 14 & 22 & 6.7 \\
15.7 & 18.2 & 2.5 & 19.3 & 26 & 15.4 \\
8.8 & 10 & 1.2 & 13.6 & 29 & 11.5 \\
11 & 14 & 3 & 11 & 22.5 & 4 \\
13.8 & 20.3 & 6.5 & 25 & 29 & 2.6 \\
20 & 22.5 & 2.5 & 14.7 & 17.4 & 3.6 \\
\hline
\end{tabular}

Mean value $=15.556621 .74$ 46.761, Standard deviation $=5.5255$ 5.476 61.2636, $\mathrm{df}=29 \mathrm{t}=60.697 \mathrm{t} 0.001 / 2=3.659$. 
Table 4 The change in amplitude and frequency of stimulus Neiguan before and after.

\begin{tabular}{|c|c|c|c|c|c|c|c|c|c|}
\hline Gender & \multicolumn{4}{|c|}{ Male } & \multicolumn{5}{|c|}{ Female } \\
\hline Vowel & \multicolumn{9}{|c|}{ i a i a i a i a } \\
\hline Change & \multicolumn{9}{|c|}{ frequency (+-) amplitude (+-) frequency (+-) amplitude (+-) } \\
\hline Age groups & \multicolumn{9}{|c|}{ Cases No. (29 cases) (18 cases) } \\
\hline 12-17 & 4 & 0 & 0 & 0 & 0 & +3 & +2 & +4 & +4 \\
\hline \multirow[t]{2}{*}{$19-30$} & 38 & +15 & +10 & +24 & +24 & +8 & +5 & +10 & +10 \\
\hline & & -2 & -2 & -2 & -2 & -1 & 0 & -2 & -2 \\
\hline \multirow[t]{2}{*}{$31-45$} & 5 & +2 & +1 & +3 & +3 & +1 & 0 & +1 & +1 \\
\hline & & -1 & -1 & 0 & 0 & 0 & 0 & -1 & -1 \\
\hline In total & & +17 & +11 & +27 & +27 & +12 & +7 & +15 & +15 \\
\hline Positive efficiency rate (\%) & & 36.17 & 23.40 & 57.44 & 57.44 & 25.53 & 14.89 & 31.91 & 31.91 \\
\hline - in total & & -3 & -3 & -2 & -2 & -2 & 0 & -3 & -3 \\
\hline Negative efficiency rate (\%) & & 6.38 & 6.38 & 4.25 & 4.25 & 4.25 & 0 & 6.38 & 6.38 \\
\hline+ In total & 47 & 20 & 14 & 29 & 29 & 14 & 7 & 18 & 18 \\
\hline Efficiency rate in total (\%) & & 42.55 & 29.78 & 61.70 & 61.70 & 29.78 & 14.89 & 8.30 & 38.30 \\
\hline Male/ female + in total & & & & & & 34 & 21 & 47 & 47 \\
\hline +Total efficiency rate (\%) & & & & & & 72.33 & 44.68 & 100 & 100 \\
\hline
\end{tabular}

3.6 Heartbeat Changes Comparison before and after Stimulating Neiguan Acupoint

Heartbeat changes experiment through stimulating Neiguan acupoint while singing proves that meridian acupoint stimulation has positive impact on heartbeat during singing pronunciation (Table 6).

\subsection{Experiment Statistics of Treatment of Disease Stimulating Acupoint during Singing}

Singing experiment adopting different acupoint stimulation combination also proves this method has the function of singing training, health rehabilitation and disease prevention (Table 7) [1].

\subsection{Deaf-Mute Rehabilitation Treatment Cases}

Case: Chen, a boy of 13 years old (born in May 29th, 2000), once in the special education school. Disease cause: Amniotic fluid choked his throat and caused Cerebral Anoxia. He lost the ability of listening and speaking. Medical history: He took ginkgo leaf tablets for a long term, and received language training but has no effect on him. Examination result: His tongue can't reach out or curl since it became stiffened, even wearing hearing aid can't improve the situation. Inspection found that his tongue is hard, not curly. Treatment: two courses of treatment (every course 6 hour/6 times/6 days). Treatment effect: He can speak and listen normally and is transferred to Ordinary normal schools.

\section{Effect Rule of Singing Meridian Comprehensive Control System}

Generally speaking, the location of the meridian can treat diseases associated with it. It can be tuned and control the pitch of singing, such as sound of high and low, strong and weak, big and small, including tone is bright and dark. Relatively, closer to acupuncture points near the center of the body, local effect is more noticeable. While stimulation of far-end acupoint [4] has obvious effect on the whole body. For instance, stimulating acupoint in the head would have strongest effect on resonance cavity in the head, and hence can be used to singing resonance adjustment and control. Stimulating acupoint in the lung has the strongest effect on breathing, and can be used to singing breathing control. The rest can be analogized in the same manner. Apart from that, the voice generated through stimulating acupoints in Yin meridian would be gentler than that came from Yang meridian. And in reverse, the voice generated from stimulating acupoint in Yang meridian would be much brighter and louder 
Table 5 The change of range of stimulus Yintang before and after.

\begin{tabular}{|c|c|c|c|c|c|c|c|c|c|c|c|}
\hline \multicolumn{12}{|c|}{ Female Male Male } \\
\hline $\begin{array}{l}\text { Without } \\
\text { stimulus range }\end{array}$ & $\begin{array}{l}\text { Stimulate } \\
\text { range }\end{array}$ & $\begin{array}{l}\text { Treble } \\
\text { comparison } \\
\text { (chromatic No.) }\end{array}$ & $\begin{array}{l}\text { Bass } \\
\text { comparison } \\
\text { (chromatic No.) }\end{array}$ & $\begin{array}{l}\text { Without } \\
\text { stimulus } \\
\text { range }\end{array}$ & $\begin{array}{l}\text { Stimulate } \\
\text { range }\end{array}$ & $\begin{array}{l}\text { Treble } \\
\text { comparison } \\
\text { (chromatic } \\
\text { No.) }\end{array}$ & $\begin{array}{l}\text { Bass } \\
\text { comparison } \\
\text { (chromatic } \\
\text { No.) }\end{array}$ & $\begin{array}{l}\text { Without } \\
\text { stimulus } \\
\text { range }\end{array}$ & $\begin{array}{l}\text { Stimulate } \\
\text { range }\end{array}$ & $\begin{array}{l}\text { Treble } \\
\text { comparison } \\
\text { (chromatic No.) }\end{array}$ & $\begin{array}{l}\text { Bass } \\
\text { comparison } \\
\text { (chromatic No.) }\end{array}$ \\
\hline$b-{ }^{\#} d^{2}$ & $f-a^{2}$ & 6 & 6 & $c^{1}-e^{2}$ & $\mathrm{f}-\mathrm{a}^{2}$ & 7 & 5 & $b-e^{2}$ & $\mathrm{f}-\mathrm{A}^{2}$ & 6 & 6 \\
\hline$b-{ }^{*} c^{2}$ & $\mathrm{~b}-{ }^{*} \mathrm{~g}^{2}$ & 6 & 6 & $\mathrm{~b}-{ }^{\#} \mathrm{f}^{2}$ & $f-b^{b}$ & 5 & 4 & $c^{1}-f^{2}$ & $a-a^{2}$ & 3 & 4 \\
\hline$c^{1}-e^{2}$ & $\mathrm{f}^{\mathrm{b}} \mathrm{b}^{2}$ & 7 & 6 & $c^{1}-f^{2}$ & $f-b^{2}$ & 7 & 5 & $c^{1}-e^{2}$ & $a-g^{2}$ & 5 & 3 \\
\hline$c^{1}-e^{2}$ & $f-a^{2}$ & 7 & 5 & $c-{ }^{1} \mathrm{e}^{2}$ & $f-b^{2}$ & 7 & 7 & $d^{1}-f^{2}$ & $\mathrm{~b}-{ }^{*} \mathrm{~g}^{2}$ & 4 & 3 \\
\hline$b-f^{2}$ & $\mathrm{f}^{\mathrm{b}} \mathrm{b}^{2}$ & 6 & 5 & $d^{1}-e^{2}$ & $\mathrm{a}{ }^{\#} \mathrm{e} 2$ & 5 & 4 & $b-f^{2}$ & $g-a^{2}$ & 4 & 4 \\
\hline$c^{1}-d^{2}$ & $\mathrm{a}-{ }^{\#} \mathrm{~g} 2$ & 3 & 5 & $b-e^{2}$ & $f-b^{2}$ & 6 & 4 & $c^{1}-e^{2}$ & $f-a^{2}$ & 7 & 5 \\
\hline$c^{2}-f^{2}$ & $g-b^{2}$ & 5 & 5 & $\mathrm{~b}-{ }^{\#} \mathrm{f}^{2}$ & $f-{ }^{b} b^{2}$ & 6 & 4 & $a-{ }^{\#} f^{2}$ & $f-a^{2}$ & 4 & 3 \\
\hline \multicolumn{12}{|c|}{$\begin{array}{l}a-{ }^{\#} f^{2} f-b^{2} 35 c^{1}-f^{2} f-a^{2} 74 b-g^{2} f-{ }^{b} b^{2} 3 \\
c^{1}-f^{2} f-b^{2} 75 b-f^{2} f-b^{2} 66 c^{1}-e^{2} f-a^{2} 75\end{array}$} \\
\hline $\begin{array}{l}\text { Mean value }=5 \\
\text { Standard differ }\end{array}$ & $\begin{array}{l}53334.733 \\
\text { nce }=1.384\end{array}$ & 3. & & & & & & & & & \\
\hline
\end{tabular}


Table 6 Heartbeat changes comparison before and after stimulating Neiguan acupoint.

\begin{tabular}{|c|c|c|c|c|c|c|c|c|c|c|c|c|c|c|c|c|c|}
\hline Basic & $\begin{array}{l}\text { Heart beat } \\
\text { (pronunciation) }\end{array}$ & $\begin{array}{l}\text { Heart beat } \\
\text { (pronunciati } \\
\text { on) }\end{array}$ & $\begin{array}{l}1 \\
2 \\
\end{array}$ & 3 & 3 & Basic & $\begin{array}{l}\text { Heart } \\
\text { beat } \\
\text { (pronun } \\
\text { ciation) }\end{array}$ & $\begin{array}{l}\text { Heart } \\
\text { beat } \\
\text { (pronunc } \\
\text { iation) }\end{array}$ & $\begin{array}{l}1 \\
2 \\
\end{array}$ & 3 & 2 & Basic & $\begin{array}{l}\text { Heart } \\
\text { beat } \\
\text { (pronun } \\
\text { ciation }\end{array}$ & $\begin{array}{l}\text { Stimulat } \\
\text { ion }\end{array}$ & $\begin{array}{l}1 \\
2 \\
\end{array}$ & 3 & 3 \\
\hline $\begin{array}{l}\text { Heart } \\
\text { beat }\end{array}$ & $\begin{array}{l}\text { Before } \\
\text { stimulation }\end{array}$ & $\begin{array}{l}\text { After } \\
\text { stimulation }\end{array}$ & $\begin{array}{l}\text { Differenc } \\
\text { e }\end{array}$ & $\begin{array}{l}\text { Differenc } \\
\text { e }\end{array}$ & $\begin{array}{l}\text { Differen } \\
\text { ce }\end{array}$ & $\begin{array}{l}\text { Heart } \\
\text { beat }\end{array}$ & $\begin{array}{l}\text { Before } \\
\text { stimulati } \\
\text { on }\end{array}$ & $\begin{array}{l}\text { After } \\
\text { stimulati } \\
\text { on }\end{array}$ & $\begin{array}{l}\text { Differe } \\
\text { nce }\end{array}$ & $\begin{array}{l}\text { Differen } \\
\text { ce }\end{array}$ & $\begin{array}{l}\text { Differe } \\
\text { nce }\end{array}$ & $\begin{array}{l}\text { Heart } \\
\text { beat }\end{array}$ & $\begin{array}{l}\text { Before } \\
\text { stimulati } \\
\text { on }\end{array}$ & $\begin{array}{l}\text { After } \\
\text { i stimulat } \\
\text { ion }\end{array}$ & $\begin{array}{l}\text { Differen } \\
\text { ce }\end{array}$ & $\begin{array}{l}\text { Differe } \\
\text { nce }\end{array}$ & $\begin{array}{l}\text { Differen } \\
\text { ce }\end{array}$ \\
\hline 1 & 2 & 3 & & & & 1 & 2 & 3 & & & & 1 & 2 & 3 & & & \\
\hline 97 & 107 & 125 & +10 & +28 & +18 & 97 & 136 & 125 & +39 & +28 & -11 & 103 & 125 & 111 & +22 & +8 & -14 \\
\hline 94 & 130 & 100 & +36 & +6 & -30 & 83 & 94 & 91 & +11 & +8 & 3 & 94 & 107 & 94 & +13 & 0 & -13 \\
\hline 86 & 95 & 86 & +9 & 0 & -9 & 81 & 100 & 103 & 19 & +22 & +3 & 75 & 79 & 91 & +4 & +6 & +12 \\
\hline 91 & 130 & 111 & +39 & +20 & -19 & 91 & 100 & 97 & +9 & +6 & -3 & 88 & 97 & 91 & +9 & +3 & -6 \\
\hline 79 & 86 & 94 & +7 & +15 & +8 & 107 & 120 & 107 & +13 & 0 & -13 & 75 & 125 & 94 & +50 & +19 & -31 \\
\hline 88 & 120 & 94 & +32 & +6 & -26 & 94 & 103 & 96 & +9 & +2 & -7 & 79 & 94 & 86 & +15 & +7 & -8 \\
\hline 88 & 111 & 120 & +23 & +32 & +9 & 83 & 100 & 111 & +17 & +28 & +11 & 83 & 103 & 86 & +20 & +3 & -17 \\
\hline 88 & 107 & 111 & +19 & +23 & +4 & 111 & 136 & 125 & +25 & +14 & -11 & 100 & 111 & 120 & +11 & +20 & +9 \\
\hline 83 & 130 & 107 & +47 & +24 & -23 & 88 & 91 & 83 & +3 & -5 & -8 & -- & -- & -- & -- & -- & -- \\
\hline 75 & 94 & 77 & +19 & +2 & -17 & 97 & 103 & 111 & +6 & +14 & -8 & -- & -- & -- & -- & -- & -- \\
\hline 94 & 115 & 107 & +2 & +13 & -8 & 86 & 107 & 21 & +21 & +17 & -4 & -- & -- & -- & -- & -- & -- \\
\hline 94 & 107 & 86 & +13 & +8 & -21 & 86 & 100 & 94 & +14 & +8 & -6 & -- & - & -- & -- & -- & -- \\
\hline 115 & 143 & 136 & +28 & +21 & -7 & 88 & 115 & 103 & +27 & +15 & -12 & --- & --- & -- & - -- & -- & - \\
\hline
\end{tabular}

(1) Mean value $=89.25$ (2) Mean value $=108.75(3)$ Mean value $=101.3$

Standard deviation $=9.959$ Standard deviation $=15.377$ Standard deviation $=14.31$.

$\mathrm{df}=35 \mathrm{t}=9.961 \mathrm{t}=7.4776 \mathrm{t}=6.160 \mathrm{t} 0.001 / 2=3.5985$.

Table 7 Training status list for persons of different voice conditions and age groups.

\begin{tabular}{|c|c|c|c|c|c|c|c|c|}
\hline Status & & & & & & & & \\
\hline $\begin{array}{l}\text { No. } \\
\text { Age group }\end{array}$ & $\begin{array}{l}\text { Acute or chronic } \\
\text {-rhinitis }\end{array}$ & $\begin{array}{l}\text { Chronic sore } \\
\text { throat }\end{array}$ & $\begin{array}{l}\text { Hoarse voice during } \\
\text { voice changing period }\end{array}$ & $\begin{array}{l}\text { Vocal hypertrophy or } \\
\text { closed is not complete }\end{array}$ & Out of tune & Normal & $\begin{array}{l}\text { Training time twice/week one } \\
\text { hour/per time (month) }\end{array}$ & $\begin{array}{l}\text { Efficiency rate } \\
100 \%\end{array}$ \\
\hline $10-18$ & & 2 & 2 & & & 5 & $2-4$ & 19.56 \\
\hline 20-35 & 4 & 2 & & 2 & 2 & 26 & $3-5$ & 78.26 \\
\hline Above 36 years old & & 1 & & & & & 3-5 & 2.17 \\
\hline
\end{tabular}

Remarks: the list is treatment statistics for vocal music learners in1993-1994. 
than that from stimulating acupoints in Yin meridian [1].

\section{Singing Meridian Comprehensive Control System Effect Overview}

Singing meridian control system has common systematic and comprehensive features [1] of singing and acupoint stimulating combination treatment, including bi-directional layout, benign comprehensiveness [4], as well as knowable, certain, observable and controllable features [4]. Therefore it can be applied to both singing study training and rehabilitation treatment of relevant diseases [1].

\section{Significance and Value of Singing Meridian Extension Mobility Research}

The significance and value of extension mobility of singing meridian lies in that it can scientifically explain the mystery of canto and singing sound mechanism, and develop scientific nature and artistry of singing theory and practice. Besides, it also created new application fields and functional category for meridian theories of traditional Chinese medicine. Research on theory and practice of singing meridian has extensive value in terms of society [2], culture, academy, science application and economic development. Meridian techniques has now been applied to other training fields such as music skill and technique training, language pronunciation skill training and speech correction, physiological and physical disease treatment, technological operation techniques during music learning process and medical rehabilitation health care fields [2].

\section{Practicing Procedures of Singing Meridian Science}

Content form, operation methods and requirement of singing meridian science includes traditional content and form of singing and meridian, while at the same time make innovations in the aspects of theory, methods, content, form and requirement of singing and meridian science. It is different from traditional singing and meridian theory content in terms of form and content. The theory, practice, operation methods and models are not limited to a single form and required changes, but focus on achieving excellent training and learning effect [1].

\subsection{Main Operation Methods}

Integration of singing meridian acupoint stimulating is a changing dynamic process of general movement of body system. Therefore, in order to adapt special requirements of singing voicing and intensify memory curative effect of disease treatment, three main stimulating operation methods are used in singing training and disease control process: fixed point stimulation, unfixed point mobile stimulation and stimulation of both fixed point and unfixed point.

Fixed point stimulation: Select acupuncture instrument needle of half cun (a unit of length $=1 / 3$ decimeter) and fix it in some part [3] of the body while singing.

Mobile stimulation on unfixed point: Select steel acupuncture needle of half cun, or a bamboo needle, magnetic acupuncture needle to stimulate some body part while singing [2].

Stimulation of both fixed acupoint and unfixed acupoint: Adopting both fixed and unfixed stimulation method while singing [2].

\subsection{Main Operation Principles}

In traditional way of singing which relies on auditory sense memory, intensified singing meridian kinetic sensory memory method which combines acupoint stimulation with fixed position, nature, location and method, and makes singing pronunciation is applied, and thus makes disease treatment effective and convenient [1].

Position fixing: Position fixing changes the features of traditional singing learning such as dynamic abstraction, uncertainty and hard to be controlled to more advanced features of singing meridian learning 
method such as being comparatively static, detailed, definite and easily to be controlled.

Determine nature makes traditional singing learning consciousness firstly govern motion descending pathway, and then govern consciousness ascending pathway transmission through adjusted sensory feelings of meridian, and provide feed-backs to multiple entirety system governed by consciousness which works in coordinated methods. Coupled with paralleled multiple memorizing function including feed-backing function of sound effectiveness from the acupoint of the body, comprehensive functions of different organs, meridians, singing and voice effects are strengthened, and thus the effect of singing sensory memory and disease rehabilitation is improved.

Acupuncture point fixing: Point fixing is the factor that has the most direct influence on singing training and treatment. Point works on faces, and faces work on parts, parts works on the whole body, the whole body impacts system transmission. Through this process, singing meridian control system can improve singing techniques during rehabilitation process. The two item works together and complement on each other.

The method is flexible, not fixed. There are actually no fixed methods and any method is subject to people. However, operation method of singing meridian control system is special in the following aspects.

Its manipulating process adopts the method of autonomic shrinkage of muscular movement and active acupoint stimulating.

Acupoint stimulation mainly adopts instruments of steel needle, bamboo needle and fingers.

Acupuncture process needs to be conducted under the guidance of instructor, other methods can be conducted by the patient.

Select and formulate appropriate singing acupoint stimulation training plan in the principle of reaching outstanding effect [2].

\section{Prospect of Singing Meridian Science Research}

Meridian application technique has very optimistic development prospect and substantive application value. It is featured by scientific system, standardization, high feasibility and great interest. It is also easy to be learned, memorized and consolidated. The technique enriches, develops teaching theories, practice method in singing, traditional medicine field, and lay solid scientific practical foundation for establishing singing theoretical system, thus providing feasible scientific grounds for research [1].

Theoretical argument is limited, while discovery in practice is indefinite. The recognition and knowledge of meridian and singing is constantly being improved. As long as we work diligently, discard disadvantages and make innovations, we can create more new models integrating various kinds of culture, and thus realize the goal of cultivating more talents at faster speed. Singing meridian science research demands our efforts, and needs to be complemented by practical application process. We will make innovations and constantly make progress to make more contributions to human culture and health industry development by using singing meridian science and meridian application techniques [2].

\section{Reference}

[1] Zhao L. 2006. Singing Meridian Study. Beijing: Beijing Science and Technology Press.

[2] Zhao L. 2014. Singing Meridian Study (Book 1). China Northeast Normal University Press.

[3] Li D. 1995. Meridian science. Shanghai: Science and Technology Press.

[4] Zhou X. D. 1995. Meridian Essence and Acupuncture Function Mechanism. Beijing: China Traditional Medicine Press.

[5] College Sports Professional Textbook Writing Committee of Henan Province. 1994. Anthropotomy. Zhengzhou: Science and Technology Press.

[6] College Sports Professional Textbook Writing Committee of Henan Province. 1994. Physiology. Zhengzhou: Science and Technology Press. 
Applying Meridian Theory into Singing Pronunciation

[7] Yang J. S. 1984. Yu Acupoint Study. Shanghai: Science and Technology Publishing Press.
[8] Zeng T. D. 2010. Meridian Science Introduction. Shanghai: Science and Technology Publishing Press. 